\title{
Data Traffic Model in Machine to Machine Communications over 5G Network Slicing
}

\author{
Mohammed Dighriri \\ Liverpool John Moorse University \\ M.H.Dighriri@2015.ljmu.ac.uk
}

\author{
Gyu Myoung Lee \\ Liverpool John Moorse University \\ G.M.Lee@ljmu.ac.uk
}

\author{
Thar Baker \\ Liverpool John Moorse University \\ T.baker@1jmu.ac.uk
}

Ali Saeed Dayem Alfoudi

Liverpool John Moores University

A.S.Alfoudi@2014.ljmu.ac.uk

\begin{abstract}
-the recent advancements in cellular communication domain have resulted in the emergence of Machine-to-Machine applications, in support of the wide range and coverage provision, low costs, and high mobility. $5 G$ network standards represent a promising technology to support the future of Machine-to-Machine data traffic. In recent years, Human-Type-Communication traffic has seen exponential growth over cellular networks, which resulted in increasing the capacity and higher data rates. These networks are expected to face challenges such as explosion of the data traffic due to the future of smart devices data traffic with various Quality of Service requirements. This paper proposes a novel data traffic aggregation model and algorithm along with a new $5 G$ network slicing based on classification and measuring the data traffic to satisfy Quality of Service for smart systems in a smart city environment. In our proposal, $5 G$ radio resources are efficiently utilized as the smallest unit of a physical resource block in a relay node by aggregating the data traffic of several Machine-to-Machine devices as separate slices based on Quality of Service for each application. OPNET is used to assess the performance of the proposed model. The simulated $5 G$ data traffic classes include file transfer protocol, voice over IP, and video users.
\end{abstract}

Keywords-Machine-to-Machine (M2M); 5G; Network Slicing; Physical Resource Block (PRB)

\section{INTRODUCTION}

Cellular networks are expected to face challenges due to the future Machine-to-Machine (M2M) data traffic with various Quality of Service (QoS) requirements such as a provision of radio resources to a large number of $\mathrm{M} 2 \mathrm{M}$ devices, prioritization, and inter-device communication [1]. The existing cellular systems might run out of capacity in the future due to significantly increasing $\mathrm{M} 2 \mathrm{M}$ traffic, resulting in the performance degradation of regular mobile traffic [1]. M2M devices transmit small and large sized data with different QoS requirements. For example, smart healthcare system devices convey big sized data that are delay sensitive [2]. The Physical Resource Block (PRB) is the smallest radio resource unit, which is allocated to a single device for data transmission in $5 \mathrm{G}$ mobile networks as shown in Figure 2. In smart systems, there are different devices transmitting numerous size of data; where some transmit small size of data thereof the capacity of the PRB is not fully used. This results in significant degradation of the system performance. This paper proposes an M2M data traffic aggregation model for efficient utilization of the $5 \mathrm{G}$ radio resources for $\mathrm{M} 2 \mathrm{M}$ communication along with $5 \mathrm{G}$ services slicing model. $5 \mathrm{G}$ radio resources will be efficiently exploited by aggregating the data of several M2M devices for each slice individually. The resources are fully utilized by isolation slice in the M2M devices to enhance the spectral effectiveness of the system.

The proposed research aims at designing and developing a novel data traffic aggregation model for $5 \mathrm{G}$ radio resources based on efficiently utilized the smallest untie of PRB by aggregating the data of several M2M devices. In addition, a new $5 \mathrm{G}$ network slicing model relies on smart systems in smart city case study, network slices will differentiate smart systems data traffic in terms of QoS requirements in each slice such as smartphones, smart traffic monitoring, and smart healthcare system.

The Optimized Network Engineering Tool (OPNET) will be used to evaluate the performance of the proposed model in term of QoS for each data traffic slices isolation. The simulated 5G data traffic classes contain File Transfer Protocol (FTP), Voice over IP (VoIP) and video users. The scenarios categorize into three slices M2M communication based on the data traffic including popular, sensitive and heavy data traffic. The results will show the proposed model's impact of M2M data traffic with different QoS characteristics of 5G networks. The network end-to-end performance will test by aggregating data of several $\mathrm{M} 2 \mathrm{M}$ devices in each slice in terms of average cell throughput, FTP average upload response time, FTP average packet end-toend delay and radio resource utilization [3].

\section{BACKGROUND}

\section{A. $5 G$ Protocols Architecture}

5G mobile network interfaces between User Equipment (UE), Evolved UMTS Terrestrial Radio Network (E-UTRAN), Evolved Packet Core (EPC) and Serving Gateways (S-GWs) are linked with protocol stacks that are used by the network component to conversion signaling messages and data [4]. The main 5G features include Carrier Aggregation (CA), Enhanced Multiple Input Multiple Output (MIMO) technology, Coordinated Multi-Point (CoMP), and Relay Node (RN) [4].

\section{B. $\quad 5 G$ Network Slicing}

$5 \mathrm{G}$ as a new generation of the mobile network is being actively discussed in the world of technology; network slicing surely is one of the most deliberated technologies nowadays. Mobile network operators such as China Mobile, and SK Telecom, and merchants such as Nokia, and Ericsson are all knowing it as a model network architecture for the coming $5 \mathrm{G}$ period [5]. This novel technology allows operators to slice one physical network among numerous, virtual, end-to-end (E2E) networks, each rationally isolated counting device, access, transport and core networks such as separating a HDD into $\mathrm{C}$ and D drives and devoted for diverse kind of services with different features and QoS requirements. each network slice, committed resources for example resources within Network Functions virtualization (NFV), Software Defined Networking (SDN), cloud computing, network bandwidth, QoS, and so on are certain as seen in Figure 1 [5]. 


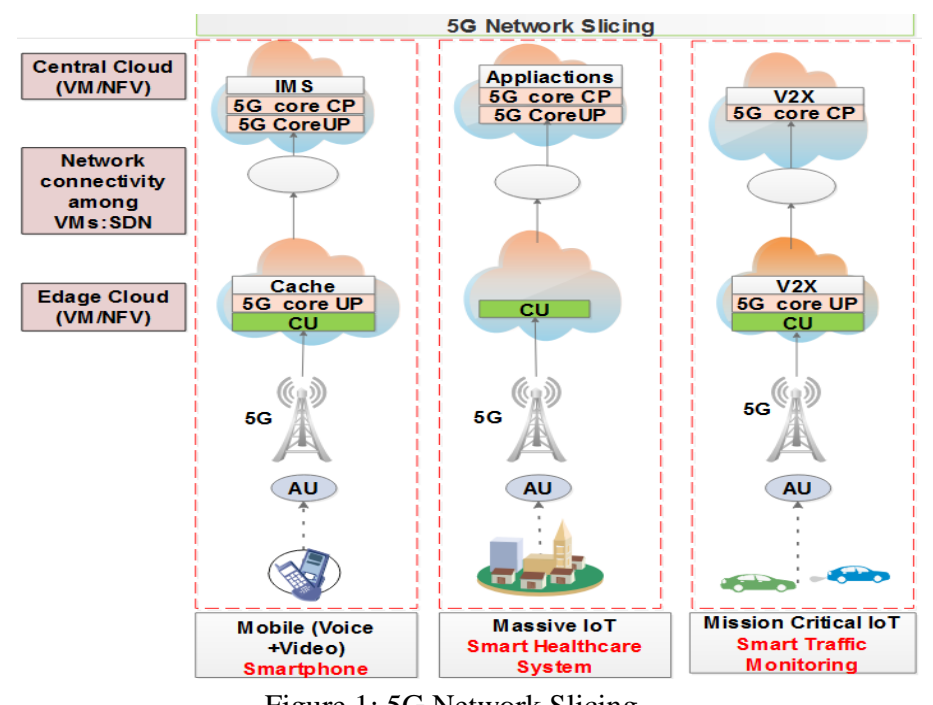

\section{Problem Statement}

Since radio resources are essential assets and scarcely available, therefore, efficient utilization is required. The novel communication technologies, such as Long Term Evolution (LTE), Long Term Evolution Advanced (LTE-A), and 5G; make use of multiple carriers schemes to offer better data rates and to ensure high QoS. The smallest resource unit allocable in the $5 \mathrm{G}$ system to an M2M device is the PRB as illustrated in Figure 2. Under favorable channel conditions, PRB is capable of transmitting several kilobytes of data. These multiple carriers' schemes are able of transmitting a large amount of data. However, in the case of M2M communication, both narrowband and broadband applications have to be considered to enhance QoS requirements. Especially, these applications have different size of data traffic, which need QoS specifications such as real-time, accuracy and priority. If one PRB is allocated to a single M2M device for data transmission of just a few bytes, then it might cause severe wastage of radio resources, also, the different type of data traffic should be considered in 5G slices approach as illustrated in Figure 3. Therefore, utilization of the full radio resources and classifying data traffic should be brilliant solutions for Data Explosion and the fairness of services in the near future.

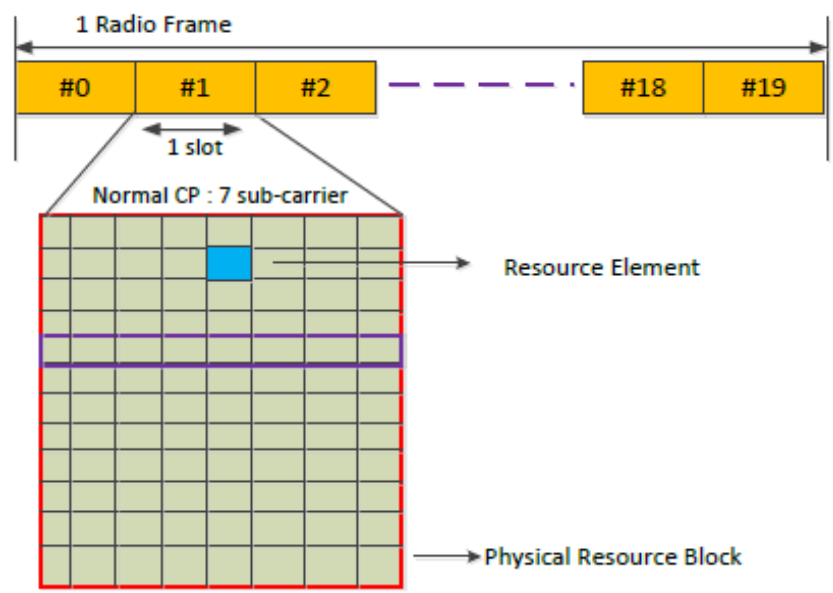

Figure 2: Physical Resource Block (PRB) [6]

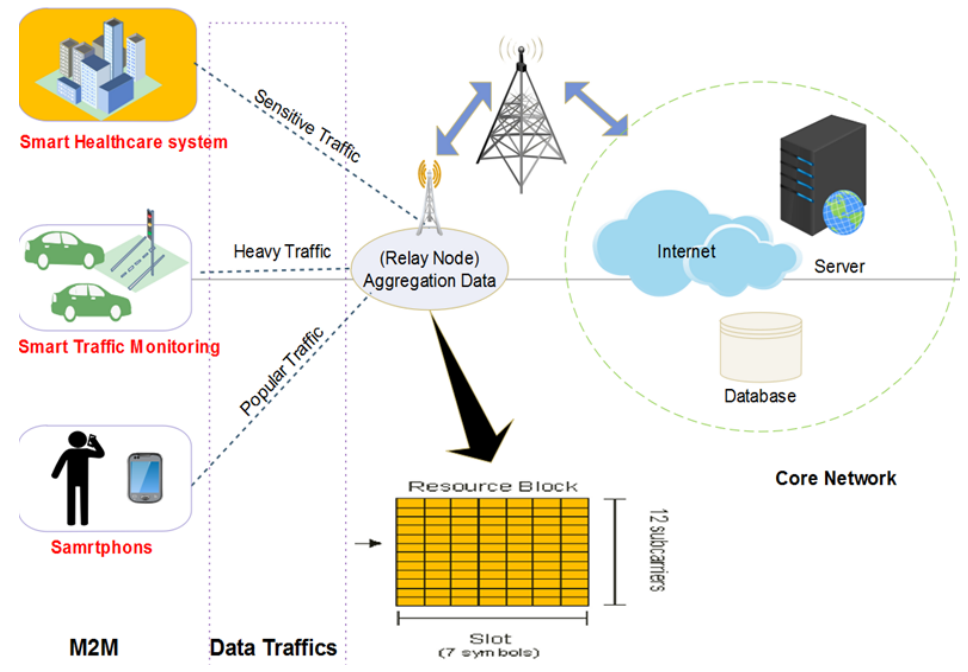

Figure 3: M2M Data Traffic on 5G Mobile Network

\section{StATE Of The ART}

\section{A. M2M Applications Data Traffic Model}

M2M communication has played a significant role in interconnecting the physical systems with the Internet world. The concept of cellular-based M2M communication is flourishing. Numerous researchers have investigated new and advanced methods to upgrade the existing cellular standards to provision M2M communication. The authors of [2] suggested that $5 \mathrm{G}$ cellular technologies as the most promising technologies for future M2M communication. The authors of [7] delivered an overview of cellular-based M2M communication and highlighted the growing M2M traffic. Traffic modeling is literally the scheming of stochastic processes, which they represent the physical parameters of the measured traffic. The initial detailed characterization of M2M has been done in [8] in which the authors gathered traffic data for seven days from one of the main cellular service providers TIER-1 in the USA. The authors determined that there is a clear difference between the features of M2M and traditional Human-Type-Communication (HTC). Their experimental results revealed that the volume of M2M traffic in uplink is much larger than in the downlink. Moreover, M2M devices generate bursty traffic as compared to the traditional mobile devices. In [9], the authors modeled the M2M traffic as a simple Poisson process due to numerous machines assigned to one server. The authors of [10] have stated that the data streams in various M2M applications follow different statistical patterns which are difficult to capture. According to [11], M2M traffic is classified into the source and aggregated traffic. Some of the available traffic models for $\mathrm{M} 2 \mathrm{M}$ are aggregated which are identified in [9] i.e., defining M2M data traffic as one stream from numerous devices. Contrarily, to model and understand the behaviour of the M2M traffic more accurately, source traffic modeling is required i.e., modeling each M2M device on its own. However, in M2M source traffic modeling, there are many challenges that need to be overcome. For example, it is very hard to model the behaviour of traffic being produced from a massive amount of devices in parallel and in the existence of strong spatial and temporal correlation between devices [12]. In [9], a Coupled Markov Modulated Poisson Processes (CMPP) Model has been 
presented, mostly aiming the source traffic modeling in $\mathrm{M} 2 \mathrm{M}$ networks.

\section{B. Limitations of Existing Models}

As per the literature review for data traffic aggregation models, limited research efforts have been done to study the impacts of data aggregation in the case of the mobile network for M2M communication. For example, authors in [9] assess the performance of data aggregation in relations to energy consumption, thus to growth the lifetime of capillary M2M networks. Though, the authors neglected the emerging mobile M2M applications in their work. Furthermore, authors in [1] suggested a system of bundling M2M data packets at the macro station also called Donor eNBs (DeNBs) to decrease the risks of congestion in backbone networks.

Therefore, this research presents a novel data aggregation model over $5 \mathrm{G}$ mobile network slices. For this purpose, based on wireless layer 3 inband $\mathrm{RN}$ is used for enhancing coverage and aggregating uplink M2M data traffic each slice separately.

\section{SYSTEM MODELS}

\section{A. $\quad$ Proposed Data Traffic Models}

Proposed models will be relying on 5G slicing networks, which will focus on classifying and measuring QoS requirement and data traffic of M2M applications such as smartphones, smart healthcare system, and smart traffic monitoring. Since M2M applications have data traffic characteristics and diverse packets size, we have developed data aggregated model in $\mathrm{RN}$ to get maximum OoS of $5 \mathrm{G}$ radio resources. Then, we have designed three data traffic classification between RN and DeNB based on Priority Queue (PQ) Approach. These two models are proposed for uplink M2M traffic in $5 \mathrm{G}$ networks.

\section{1) M2M Data Aggregation Model}

The proposed model is relying on aggregating data from several M2M devices at the Packet Data Convergence Protocol (PDCP) layer of the RN. The PDCP layer performs header compression, delivery, and retransmission of PDCP Session Data Units (SDUs), duplicate detection, etc. In the proposed model, PDCP layer is used for the aggregation of the M2M data in uplink. The main reason for selecting PDCP for aggregation in the uplink is to aggregate data with a minimum number of the additional headers.

The individual data packets from the several M2M devices approach the PHY layer of aggregation device with various intact headers such as Medium Access Control (MAC), Radio Link Control (RLC), and PDCP. The headers are removed as the received data is transported to the upper layers. On arriving the PDCP, all the headers are removed and only the payload from the individual devices are available which are aggregated. One single aggregation buffer $B$ at the RN is considered to aggregate M2M traffic. This buffer aggregates data from different M2M devices ensuring QoS for both the $5 \mathrm{G}$ and M2M traffic. In this implementation, RN is used for M2M devices and DeNB for $5 \mathrm{G}$ traffic. In order to reach the maximum performance improvements in spectral efficiency, packet propagation delay, and cell throughput, we consider scenarios in which all the M2M devices communicate with the DeNB through a RN. The M2M data aggregation algorithm is shown in Figure 4 and described as follows:
- Data from $K \mathrm{M} 2 \mathrm{M}$ devices is considered for aggregation.

- The essential parameter for M2M data aggregation is the maximum delay time Tmax for the packet at the RN.

The maximum delay time Tmax is an essential parameter for M2M data and is calculated according to the various traffic classes of the M2M devices. M2M data have different priorities according to their applications. For example, data packets received from the $\mathrm{M} 2 \mathrm{M}$ device deployed in a smart healthcare system scenario for the measurement of temperature or pulse rate of the patient have high priority over the packets from $\mathrm{M} 2 \mathrm{M}$ devices which are deployed in smartphones. The data packets from a device having the highest priority face the smallest delay. Therefore, we initiate the Tmax value as the inter-send time of the M2M device data with the highest priority. For example, in the simulation setup for distinct M2M applications, the intersend time of the M2M traffic models is $1 \mathrm{~ms}$, which is the maximum time a packet is delayed at the RN. Thus, the value of the Tmax is initiated as $1 \mathrm{~ms}$, which means that the data packets received from the distinct $\mathrm{M} 2 \mathrm{M}$ devices are delayed for $1 \mathrm{~ms}$ at the RN.

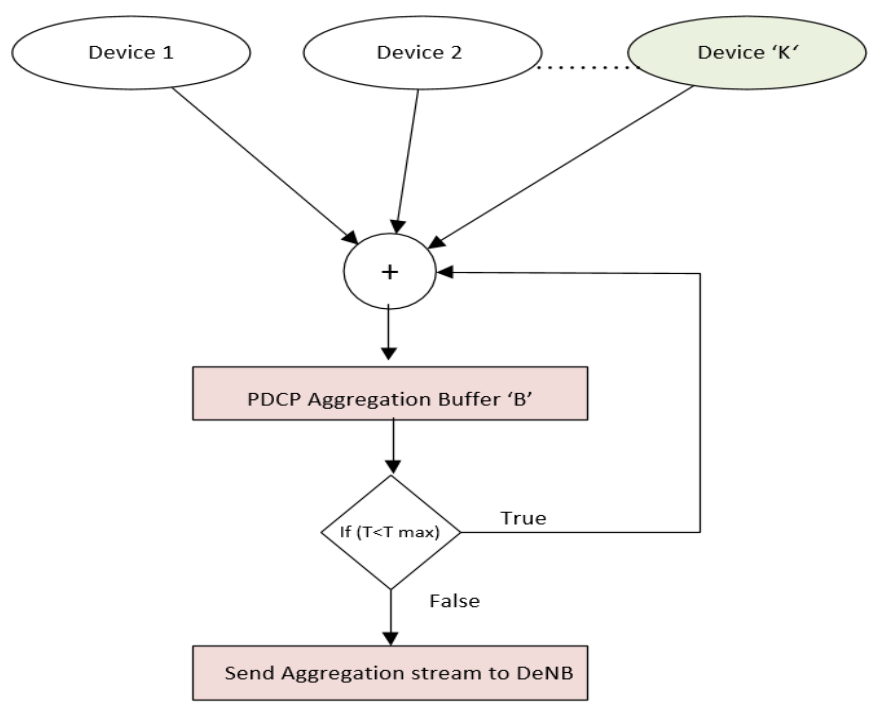

Figure 4: M2M data aggregation algorithm

The value of Tmax is adaptive, i.e., the algorithm updates the value of Tmax if RN receives packets from a device, which has higher priority than the priorities of all the other device in the queue of the RN. The data from all the M2M devices is buffered at the RN. The individual IP headers of all the M2M devices are kept intact. The data packets are buffered until time delay approaches Tmax. In order to compare the performance of data aggregation model in narrowband and broadband $\mathrm{M} 2 \mathrm{M}$ application scenarios, the aggregation scale for M2M devices is kept 1 (un-aggregated), 5, 10, 15 and 20 in both cases. The aggregation scale represents the number of devices, which are aggregated. For example, in a scenario with $180 \mathrm{M} 2 \mathrm{M}$ devices, the aggregation scale of 5, 10, 15 and 20 means that the data from the group of 5, 10, 15 and 20 devices is aggregated at the RN respectively. The aggregated data is sent to the DeNB through the $U n$ interface where the data is de-multiplexed. The individual IP streams are then sent to the respective application server by the DeNB.

The M2M packets flow from the M2M devices to the Access Gateway (aGW) through $\mathrm{RN}$ is described in Figure 5. $K$ number 
of M2M devices transmits data packets to the RN, which are collected at the PHY layer of the RN. The packets are transported to the PDCP layer of the RN on the uplink. The IP packets are packed according to their Quality Control Identifier (QCI) values in the aggregation buffer. The aggregation buffer collects packets from several M2M devices. The data packets are placed in the aggregation buffer according to the packet arrival from the different devices. The detailed structure of the aggregated data stream is depicted in Figure 5 where only the layer two protocols are presented to illustrate the aggregation of the M2M data. The RN PHY layer receives the data packets in the form of distinct Transport Block Size (TBS). The TBS are shown from 1 to $K$, which shows the TBS transmitted by the M2M devices at the RN. The data packets arrive the RLC through MAC layer. The RLC headers are removed and remaining Protocol Data Unit (PDU) is transported to the PDCP. The received PDUs at the PDCP layer comprised of the individual IP headers of each M2M device and pack into single PDCP buffer.

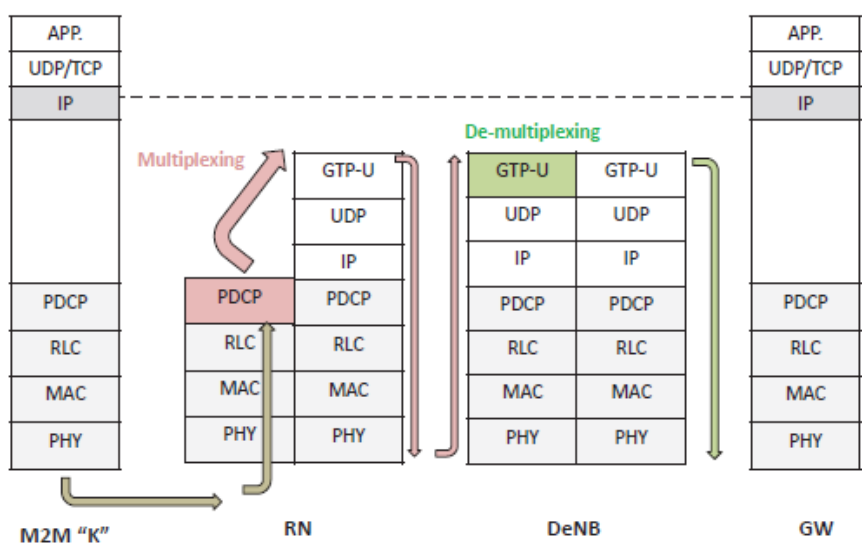

Figure 5: M2M data packets flow diagram [9]

\section{B. Design a New $5 G$ Network Slicing Model}

The 5G wireless network terminal offers exceptional QoS through a diversity of networks. Nowadays, the mobile Internet users choose manually the wireless port of different Internet service providers (ISP) without having the opportunity to exploit the QoS history to choose the suitable mobile network linking for a provided service. In the future, the $5 \mathrm{G}$ phone will deliver an opportunity for QoS analysis and storage of measured data traffic in the mobile network terminal. There are diverse QoS parameters (e.g., bandwidth, delay, jitter, and reliability) which will support in future of $5 \mathrm{G}$ mobile running in the mobile terminal. System processes will offer the best appropriate wireless connection based on needed QoS automatically. Therefore, we will consider different types of data traffic (e.g., sensitive, popular, and heavy traffic) as service slices model as shown in Table 1 [13]. These data traffic types will be working as following M2M communication environment.

\section{1) Smartphones}

Smartphones and tablets with important recent technology are represented as popular data traffic. Although smartphones are expected to continue as the key personal device and more develop in terms of performance and ability, the number of personal devices growth was driven by such devices as wearable or sensors to reach millions in 2020. In these devices the content type of mobile streaming is video; the total of the flow packets is regularly several megabytes or even tens of megabytes, it is of many packets; the transmission way is usually continual transmission; the priority is generally low because the video needs broad bandwidth, and likely blocked in congestion [14].

\section{2) Smart Healthcare System}

The smart healthcare system as sensitive data traffic is a promising model, which has currently achieved extensive attention in research and industry. A sensor Body-AreaNetwork (BAN) is generally positioned around the patient to gather information about the numerous health parameters, for instance blood pressure, pulse rate, and temperature. Moreover, the patients are also monitored repeatedly by placing smart M2M sensors on the body of the patient when they are outside the hospitals or home. For handling critical situations, alarms are triggered to send messages to the related physicians for urgent treatment [11]. In a smart healthcare system scenario, in order to monitor the patients frequently outside the medical centres (e.g., hospitals) the patients are equipped with smart $\mathrm{M} 2 \mathrm{M}$ devices that monitor various health parameters.

\section{3) Smart Traffic Monitoring}

Smart traffic monitoring allows the conversation of alerted information between vehicles infrastructure and the system applications over communication approaches and technologies. In this system we will consider heavy data traffic. Vehicles communicate with other Vehicles (V2V) or communicate with smart traffic monitoring servers, Vehicle to Infrastructure (V2I). This system application includes the collision avoidance and safety, parking time, the Internet connectivity, transportation time, fuel consumption, video monitoring, etc. [15]. In the case of emergency, the information from devices positioned to monitor emergency situations is transmitted to other networked vehicles within the communication range. To prevent any more accidents, the communication between the server and vehicles should be very fast for the detection of emergency messages and delivery of alerting messages. Since the response time of the warning messages is very small, collision avoidance services request the high level of QoS services and low latency can be supported by the $5 \mathrm{G}$ cellular networks. According to [15], the alerting messages are small in size and must only be sent in critical circumstances for effective using of the communication network bandwidth. Traffic and infrastructure management play an important role in controlling the issue of traffic congestion.

Table 1:5G services requirements

\begin{tabular}{|l|l|c|c|c|c|}
\hline Services & $\begin{array}{l}\text { Traffic } \\
\text { types }\end{array}$ & Priority & Latency & $\begin{array}{l}\text { Minimum } \\
\text { bandwidth }\end{array}$ & Mobility \\
\hline Smartphones & Popular & $10 \mathrm{~ms}$ & High & $100 \mathrm{kbps}$ & Yes \\
\hline $\begin{array}{l}\text { Smart } \\
\text { healthcare } \\
\text { system }\end{array}$ & Sensitive & $1 \mathrm{~ms}$ & Low & $100 \mathrm{Mbps}$ & Yes \\
\hline $\begin{array}{l}\text { Smart traffic } \\
\text { monitoring }\end{array}$ & Heavy & $5 \mathrm{~ms}$ & Low & $25 \mathrm{Mbps}$ & Yes \\
\hline
\end{tabular}




\section{DATA TRAFFIC SLICES MODEL}

\section{A. Data Traffic Slices Algorithm}

In the data traffic slices model, we will consider associating our previous data traffic aggregation model, which was enhancing QoS by efficient utilization of the $5 \mathrm{G}$ radio resources for $\mathrm{M} 2 \mathrm{M}$ and principle idea of PQ approach. These smart devices have various of priorities queuing which depends on the priority of the packets, the highest priority is transmitted on the output port first and then the packets with lower priority and so on as illustrated in data traffic slices algorithm in Figure 6 [16]. Therefore, we design our smart systems environment in three level of priorities high (slice1), medium (slice2), and low (slice3), rely on the data traffic types as following:

- Smart healthcare system as sensitive data with high priority (1ms)

- Smart traffic monitoring as heavy data with medium priority (5ms)

- Smartphones as popular data with low priority (10ms)

As shown in Table 1, these data traffic will work in a form of slicing over the $5 \mathrm{G}$ mobile network in the uplink path between $\mathrm{RN}$ and DeNB based on user plane interface as shown in Figure 7.

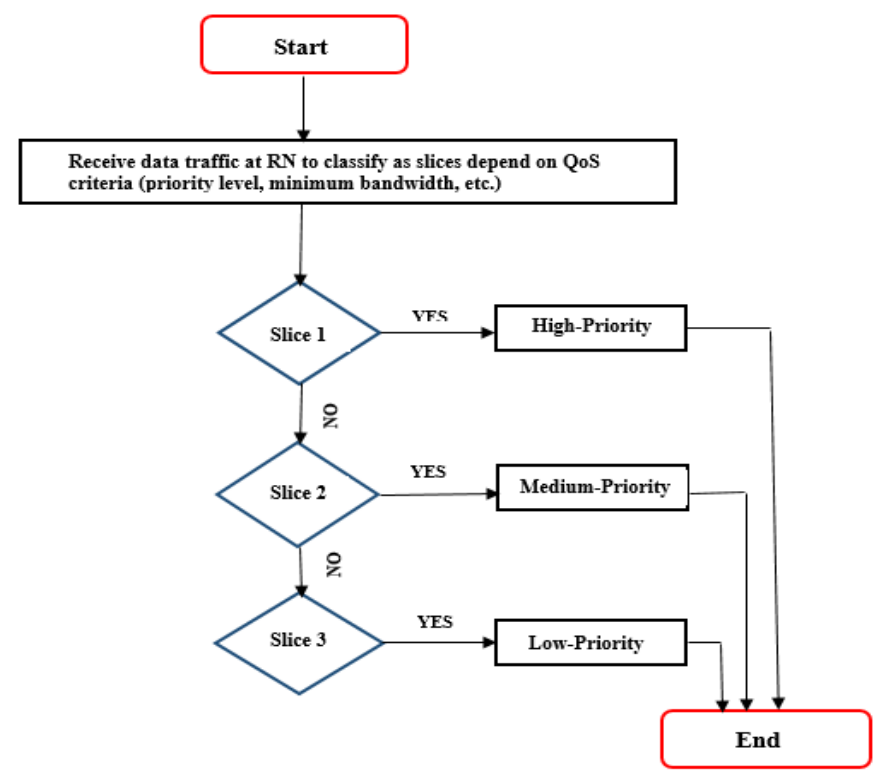

Figure 6: Data traffic slices algorithm

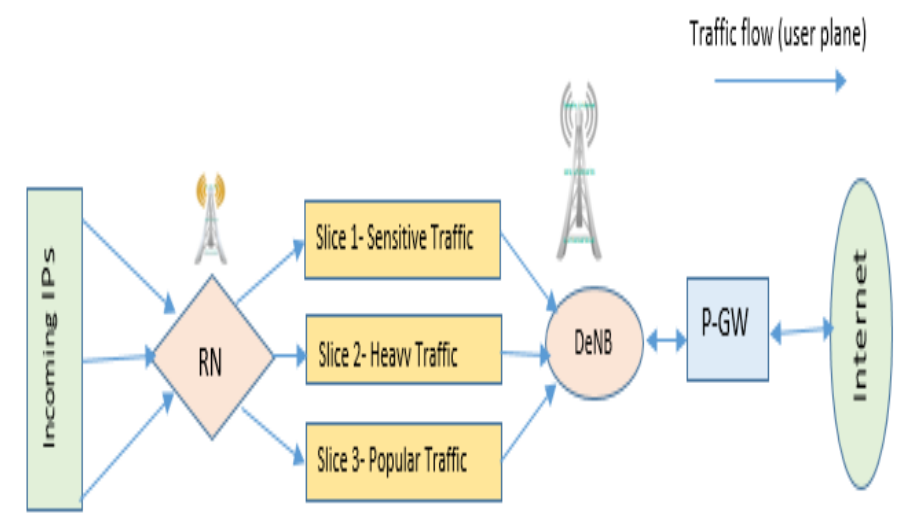

Figure 7: Data traffic slices proposed model

\section{B. QoS in Slices}

QoS could be considered as the capability of the network to provision decent services in order to accept good users. In other words, QoS considers the level of user satisfactions and network performance. Applications such as smartphones in our case study have different protocols for example HTTP, FTP, and e-mail are not sensitive to delay of transferred information to assess QoS in significant problems, whereas other applications such as smart healthcare system have a number of protocols for example video and voice are more sensitive to loss, delay and jitter of the information. Consequently, our smart systems for QoS provision considers several protocols like FTP, VoIP. Furthermore video users are requiring an essential attention to ensure that these IPs packets are not delayed or lost while be transmitted over the network [17]. VoIP QoS can be measured according to ITU recommendations based on different parameters (e.g., delay, jitter, and packet loss), these parameters can be altered and controlled within the suitable range to enhanced VoIP, FTP, and video QoS requirements. Parameters affecting QoS are briefly shown in the following sub-sections [17]:

1) Latency

As a delay sensitive application such as smart healthcare system, it has voice, file and, video cannot sustain too much delay. Latency is the average time it takes for a packet to convey from its source to its destination. A patient who has sensors to control and track up his/her condition, sensor sources such as voice and video should be reached the end destination in a few milliseconds without delay. Moreover, the delay should keep as low as possible but if there is too much traffic on uplink side of $5 \mathrm{G}$ mobile network that could cause congestions, or if there is a call a voice packet gets stuck behind a bunch of data packets such as an email attachment, the voice packet will be delayed to the point where the quality of the call is compromised [18]. The maximum amount of latency that a voice call can tolerate one way is 150 Milliseconds $(0.15 \mathrm{sec})$ but in our case study it is considered to be 10 Milliseconds $(0.1 \mathrm{sec})$ for the sensitive application system.

Equation (1) illustrates the calculation of delay where Average delay (D) is expressed as the sum of all delays $(d i)$, divided by the total number of all measurements $(N)$ [19].

$$
D=\sum_{i=1}^{N} d i / N
$$

\section{2) Jitter (Variation of Delay)}

In order to be understandable, for example VoIP, voice packets should arrive at regular Intervals. Jitter defines the degree of fluctuation in packet access, which could be affected by too much traffic on the line [19]. Voice packets can sustain only about 75 Milliseconds $(0.075 \mathrm{sec})$ but is preferred to be 40 Milliseconds $(0.040 \mathrm{sec})$ of jitter delay [17].

Equation (2) illustrates the calculation of jitter (j). Both average delay and jitter are measured in seconds. Clearly, if all ( $d i)$ delay values are equal, then $D=d i$ and $J=O$ (i.e., there is no jitter) [17]. 


$$
J=\sqrt{\frac{1}{N-1} \sum_{i=0}^{n}(d i-D)^{2}}
$$

\section{3) Packet loss}

Packet loss is the term used to refer to the packets that do not arrive at the purposed destination that occurred when a device such as switch, link, and router is overloaded and cannot accept any incoming data at a given moment [16]. Packets will be dropped during periods of network congestion. For example, voice traffic can sustain less than a $3 \%$ loss of packets $(1 \%$ is optimum) before callers feel at gaps in conversation [19].

Equation (3) illustrates the calculation of packet loss ratio defined as a ratio of the number of lost packets to the total number of transmitted packets Where $N$ equals the total number of packets transmitted during a specific time period, and $N L$ equals the number of packets lost during the same time period [17].

$$
\text { Loss packets ratio }=\left(N_{L} / N\right) \times 100 \%
$$

\section{Simulation Environment}

The simulation tool implemented in this research proposal is OPNET version 17.5. OPNET is an object-orientated simulation tool for creation network modeling and QoS analysis of the simulation of network communication, network devices, and protocols. Therefore, we will consider smart systems which consist of different priorities based on data traffic types in each slice such as slice one with high priority, slice two with medium priority, and slice three with low priority. We will also prepare different applications sources VoIP, FTP, and video to measure QoS such as latency, Jitter, and packet loss for each slice [16]. The performance of the proposed models will be evaluated by three scenarios. In the first scenario, M2M data packets are relayed in uplink without multiplexing. In the second scenario, the data packets from all the active M2M devices which are positioned in the nearness of the $\mathrm{RN}$ are aggregated at the $\mathrm{RN}$ before being sent to the DeNB. Though, only the periodic perhop control model is used in which the large aggregated data packets are served to guarantee full utilization of PRBs. In the third scenario, an expiry timer is introduced in order to limit the multiplexing delay especially in the low loaded scenarios between RN and DeNB. In this situation, the aggregated packet is served after Tmax at the latest. All the overhead stated scenarios are further sub-categorized into numerous subscenarios. In the first sub-scenario, $200 \mathrm{M} 2 \mathrm{M}$ devices are placed in the nearness of the RN. The number of M2M devices is incremented by 200 in the subsequent sub-scenarios.

\section{CONCLUSION}

We proposed M2M data traffic aggregation model and algorithm in fixed RNs for uplink in $5 \mathrm{G}$ cellular networks. It improves the radio resource utilization for $\mathrm{M} 2 \mathrm{M}$ commutations in $5 \mathrm{G}$ networks. It offers a maximum multiplexing gain in PDCP layer for data packets from the several M2M devices along with considering diverse priorities to solve packets E2E delay. Further, this research is proposed data traffic slicing model and algorithm for classifying and measure QoS requirement, it is based upon smart systems in smart city case study to support and assist the operations of diverse systems such as smart traffic monitoring, smart healthcare system, and smartphones. In the future work, we will reveal the simulation results and analysis the proposed data traffic slicing model in different data traffic scenarios such as sensitive, popular, and heavy traffics. The proposed models can be offered opportunities for the future researchers in term of resolving data traffic explosion and fairness of services area.

\section{REFERENCES}

[1] M. Iftikhar, B. Landfeldt, S. Zeadally, and A. Zomaya, "Service level agreements (SLAs) parameter negotiation between heterogeneous 4G wireless network operators," Pervasive Mob. Comput., vol. 7, no. 5, pp. 525-544, 2011.

[2] S. Y. Lien and K. C. Chen, "Massive access management for QoS guarantees in 3GPP machine-to-machine communications," IEEE Commun. Lett., vol. 15, no. 3, pp. 311-313, 2011.

[3] M. Hasan, E. Hossain, and D. Niyato, "Random access for machineto-machine communication in LTE-advanced networks: Issues and approaches," IEEE Commun. Mag., vol. 51, no. 6, pp. 86-93, 2013.

[4] I. Abdalla and S. Venkatesan, "Remote subscription management of M2M terminals in 4G cellular wireless networks," in Proceedings Conference on Local Computer Networks, LCN, 2012, pp. 877-885. M. Tesanovic and M. Nekovee, "mmWave-Based Mobile Access for 5G: Key Challenges and Projected Standards and Regulatory Roadmap," 2015 IEEE Glob. Commun. Conf., pp. 1-6, 2015. 3GPP, "LTE; Evolved Universal Terrestrial Radio Access (EUTRA); Physical channels and modulation (3GPP TS 36.211 version 8.9.0 Release 8)," ETSI TR 136 311, pp. 1-85, 2010.

[7] K. C. Chen and S. Y. Lien, "Machine-to-machine communications: Technologies and challenges," Ad Hoc Networks, vol. 18, pp. 3-23, 2014.

[8] A. Gohil, H. Modi, and S. K. Patel, "5G technology of mobile communication: A survey," in 2013 International Conference on Intelligent Systems and Signal Processing, ISSP 2013, 2013, pp. 288-292.

[9] F. Ghavimi and H. H. Chen, "M2M communications in 3GPP LTE/LTE-A networks: Architectures, service requirements, challenges, and applications," IEEE Commun. Surv. Tutorials, vol. 17, no. 2, pp. 525-549, 2015.

[10] G. Casale, E. Z. Zhang, and E. Smirni, "Trace data characterization and fitting for Markov modeling," Perform. Eval., vol. 67, no. 2, pp. 61-79, 2010.

[11] M. Chen, J. Wan, and F. Li, "Machine-to-machine communications: Architectures, standards and applications," KSII Transactions on Internet and Information Systems, vol. 6, no. 2. pp. 480-497, 2012.

[12] X. Jian, X.-P. Zeng, Y.-J. Jia, J.-Y. Yang, and Y. He, “Traffic modeling for machine type communication and its overload control," Tongxin Xuebao/Journal Commun., vol. 34, no. 9, pp. 123-131, 2013.

[13] C. S. Lee, G. M. Lee, and W. S. Rhee, "Smart Ubiquitous Networks for future telecommunication environments," Comput. Stand. Interfaces, vol. 36, no. 2, pp. 412-422, 2014.

[14] A. Annunziato, "5G vision: NGMN - 5G initiative," in IEEE Vehicular Technology Conference, 2015, vol. 2015.

[15] M. J. Booysen, S. Zeadally, and G.-J. van Rooyen, "Survey of media access control protocols for vehicular ad hoc networks," IET Commun., vol. 5, no. 11, pp. 1619-1631, 2011.

[16] G. Zirong and Z. Huaxin, "Simulation and analysis of weighted fair queueing algorithms in OPNET," in Proceedings - 2009 International Conference on Computer Modeling and Simulation, ICCMS 2009, 2009, pp. 114-118.

[17] L. Ji, X. Yin, X. Shi, and Z. Wang, "Conversational model based VoIP traffic generation," in 3rd International Conference on Networking and Services, ICNS 2007, 2007.

[18] K. Nisar, A. M. Said, and H. Hasbullah, "Enhanced performance of packet transmission using system model over VoIP network," in Proceedings 2010 International Symposium on Information Technology - Engineering Technology, ITSim'10, 2010, vol. 2, pp. 1005-1008.

[19] Y. Zhang, Z. Li, S. Mei, L. Xiao, and M. Wang, "A new approach for accelerating IPSec communication," in 1st International Conference on Multimedia Information Networking and Security, MINES 2009, 2009, vol. 2, pp. 482-485. 